\title{
Vaginal microbiome modulates topical antiretroviral drug pharmacokinetics
}

\author{
Ekaterina Taneva, ${ }^{1}$ Shada Sinclair, ${ }^{2}$ Pedro M.M. Mesquita, ${ }^{2}$ Brian Weinrick, ${ }^{1}$ Scott A. Cameron, ${ }^{3}$ \\ Natalia Cheshenko, ${ }^{2}$ Kerry Reagle, ${ }^{4}$ Bruce Frank, ${ }^{4}$ Sujatha Srinivasan, ${ }^{5}$ David Fredricks, ${ }^{5}$ \\ Marla J. Keller, ${ }^{6}$ and Betsy C. Herold ${ }^{1,2}$ \\ 'Department of Microbiology \& Immunology, ${ }^{2}$ Department of Pediatrics, and ${ }^{3}$ Department of Biochemistry, Albert Einstein \\ College of Medicine, Bronx, New York, USA. ${ }^{4}$ Particle Sciences, Inc., Bethlehem, Pennsylvania, USA. ${ }^{5}$ Vaccine and Infectious \\ Disease Division, Fred Hutchinson Cancer Research Center, University of Washington, Seattle, Washington, USA. \\ ${ }^{6}$ Department of Medicine, Albert Einstein College of Medicine, Bronx, New York, USA.
}

\begin{abstract}
Tenofovir gel and dapivirine ring provided variable HIV protection in clinical trials, reflecting poor adherence and possibly biological factors. We hypothesized that vaginal microbiota modulates pharmacokinetics and tested the effects of $\mathrm{pH}$, individual bacteria, and vaginal swabs from women on pharmacokinetics and antiviral activity. Tenofovir, but not dapivirine, uptake by human cells was reduced as $\mathrm{pH}$ increased. Lactobacillus crispatus actively transported tenofovir leading to a loss in drug bioavailability and culture supernatants from Gardnerella vaginalis, but not Atopobium vaginae, blocked tenofovir endocytosis. The inhibition of endocytosis mapped to adenine. Adenine increased from $65.5 \mu \mathrm{M}$ in broth to $246 \mu \mathrm{M}$ in Gardnerella, but decreased to $9.5 \mu \mathrm{M}$ in Atopobium supernatants. This translated into a decrease in anti-HIV activity when Gardnerella supernatants or adenine were added to cultures. Dapivirine was also impacted by microbiota, as drug bound irreversibly to bacteria, resulting in decreased antiviral activity. When drugs were incubated with vaginal swabs, $30.7 \% \pm 5.7 \%$ of dapivirine and $63.9 \% \pm 8.8 \%$ of tenofovir were recovered in supernatants after centrifugation of the bacterial cell pellet. In contrast, no impact of microbiota on the pharmacokinetics of the prodrugs, tenofovir disoproxil fumarate or tenofovir alafenamide, was observed. Together, these results demonstrate that microbiota may impact pharmacokinetics and contribute to inconsistent efficacy.
\end{abstract}

Authorship note: ET and SS contributed equally to this work.

Conflict of interest: The authors have declared that no conflict of interest exists.

Submitted: December 28, 2017 Accepted: May 31, 2018 Published: July 12, 2018

\section{Reference information:} JCI Insight. 2018;3(13):e99545. https://doi.org/10.1172/jici. insight.99545.

\section{Introduction}

Tenofovir (TFV) gel and a dapivirine (DPV) intravaginal ring (IVR) have been evaluated in efficacy trials for preexposure prophylaxis (PrEP). A 39\% reduction in HIV and 51\% reduction in herpes simplex virus-2 (HSV-2) acquisition were observed with pericoital dosing of $1 \%$ vaginal TFV gel in a phase $2 b$ trial $(1,2)$. However, daily or pericoitally dosed TFV gel was not protective in 2 subsequent phase 3 trials $(3,4)$. A DPV IVR provided $27 \%$ and $31 \%$ HIV protection, respectively, in 2 trials, with greater protection observed in women older than 21 years (56\%; 95\% CI [31 to 71], $P<0.001)$ compared with younger participants $(-27 \%$ $[-133$ to 31$], P=0.45)(5,6)$. Poor adherence contributed to the outcomes in all of the studies, but even with high adherence, protection was incomplete, suggesting a role for biological factors $(3,5,7)$.

The efficacy of topical PrEP is impacted by tissue permeability, transport into and out of cells, metabolism, and stability in the complex and dynamic mucosal environment. We previously showed that TFV enters human female genital tract (FGT) epithelial and immune cells by endocytosis, reflecting little or no organic anion transporter (OAT) expression (8-10). In contrast, the prodrugs TFV disoproxil fumarate (TDF) and TFV alafenamide (TAF) passively diffuse into cells, where they are metabolized by intracellular enzymes to TFV $(10,11)$. The differences in transport mechanisms are reflected by their relative potency; TDF and TAF inhibit HIV-1 and HSV-2 in human cell or explant tissue and murine models at 100-fold lower concentrations than TFV (10-15). Once inside a cell, TFV is phosphorylated by kinases to tenofovir diphosphate (TFV-DP), which has a prolonged intracellular half-life and competes with cellular 2'-deoxyadenosine triphosphate (dATP) for incorporation into the HIV DNA chain during reverse transcription or into HSV DNA during replication $(16,17)$. In contrast, DPV, which passively diffuses into and out of cells, does not require intracellular modifications and has a short intracellular half-life. 
A
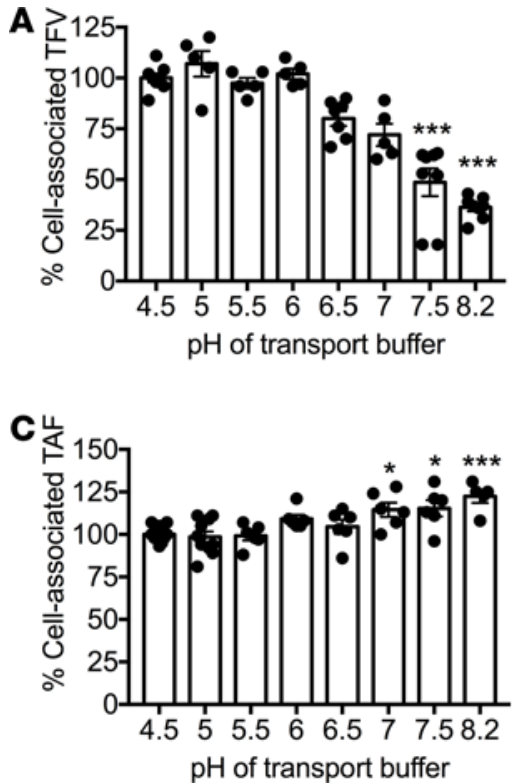

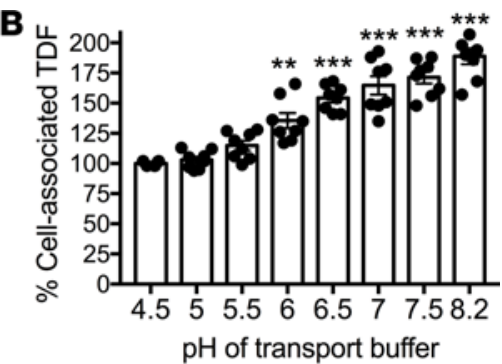

D

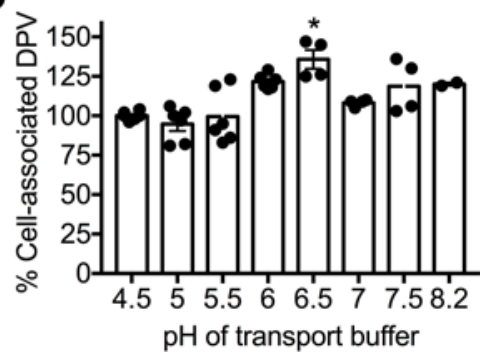

Figure 1. Impact of pH on drug uptake by human cells. Jurkat cells were exposed to radiolabeled TFV $(1 \mu \mathrm{M})(\mathbf{A})$, $\operatorname{TDF}(1 \mu \mathrm{M})(\mathbf{B}), \operatorname{TAF}(0.1 \mu \mathrm{M})(\mathbf{C})$, or DPV $(1 \mu \mathrm{M})$ (D) for 45 minutes in transport medium at the indicated $\mathrm{pH}$ and cell-associated radioactivity quantified by scintillation counting. Data are expressed as percentage of uptake at $\mathrm{pH} 4.5$ and represent mean \pm SEM of 3 independent experiments conducted in duplicate. Statistical significance was assessed using 1-way ANOVA with Bonferroni's post hoc test relative to uptake at $\mathrm{pH} 4.5 .{ }^{*} P<0.05$; ${ }^{* *} P<0.01$, ${ }^{* * *} P<0.001$

While the role of gut bacteria in drug metabolism is well established (18), only recently has the potential for vaginal microbiota to modulate drug pharmacokinetics (PK) been recognized. A secondary analysis of the CAPRISA 004 TFV gel study found that efficacy was higher in women with a lactobacillusdominant microbiome compared with women with a more diverse microbial community and suggested that Gardnerella vaginalis actively metabolized tenofovir (19). Similarly, a study comparing safety and PK of 6 daily applications of TFV vaginal gel or film found that cervical tissue TFV-DP levels were higher in women having a Nugent score 0-3 (reflecting lactobacillus predominance) but were lower in women with higher $G$. vaginalis concentrations (20). To further evaluate the potential impact of the microbiome on vaginal $\mathrm{PrEP}$ and to explore mechanisms whereby microbiota might modulate drug PK, we compared the effects of $\mathrm{pH}$, individual bacterial species, bacterial culture supernatants, and vaginal swab eluates on the intracellular accumulation and anti-HIV activity of TFV, TDF, TAF, and DPV.

\section{Results}

Impact of extracellular $\mathrm{pH}$ on drug uptake. Vaginal $\mathrm{pH}$ in healthy, reproductive-age women with a Lactobacillus crispatus - or Lactobacillus jensenii-dominant microbiome is typically between 3.8 and 4.5, but increases when acid-producing lactobacilli are less prevalent, such as in the setting of bacterial vaginosis (BV) or following exposure to semen, when $\mathrm{pH}$ increases to $7-8.2$ for several hours (21). Cervical $\mathrm{pH}$ is typically higher in the range of 6.0-7.3 (22). Optimally, drug PK should not be impeded by changes in $\mathrm{pH}$. To test the effects of $\mathrm{pH}$ on drug uptake, Jurkat $\mathrm{T}$ cells were exposed to radiolabeled drugs in transport medium at $\mathrm{pH}$ 4.5-8.2. Relative to the intracellular accumulation of radiolabeled drug at $\mathrm{pH} 4.5$, TFV decreased whereas TDF increased as the $\mathrm{pH}$ of the culture medium was raised. In contrast, $\mathrm{pH}$ had little or no impact on DPV or TAF (Figure 1).

Impact of bacteria on TFV and DPV PK. To evaluate whether exposure of drugs to individual bacterial species resulted in any change in drug bioavailability, radiolabeled TFV, TDF, or TAF $(1 \mu \mathrm{M})$ was incubated with approximately $1 \times 10^{9}$ colony forming units (CFU) of L. crispatus, L. jensenii, Lactobacillus iners, or G. vaginalis. The bacteria were then pelleted by centrifugation and the radioactivity recovered in the culture supernatant was quantified as a biomarker of drug available for uptake by human cells. Repeated measures showed little variability in scintillation counts. There was an approximately $50 \%-75 \%$ reduction in the amount of TFV recovered following incubation with live, but not heat-inactivated L. crispatus (strains 60, SJ-3C, and M35) (Figure 2, A and C). In contrast, there was little impact on recovery of TFV following incubation with the other bacterial species including multiple isolates of $G$. vaginalis even with more prolonged drug exposure (Figure 2A and Supplemental Figure 1; supplemental material available online with this article; https://doi. org/10.1172/jci.insight.99545DS1). None of the bacteria had significant effects on the recovery of TDF or TAF (Figure 2A). Similar results were obtained when $L$. crispatus or $G$. vaginalis was incubated with unlabeled 
A
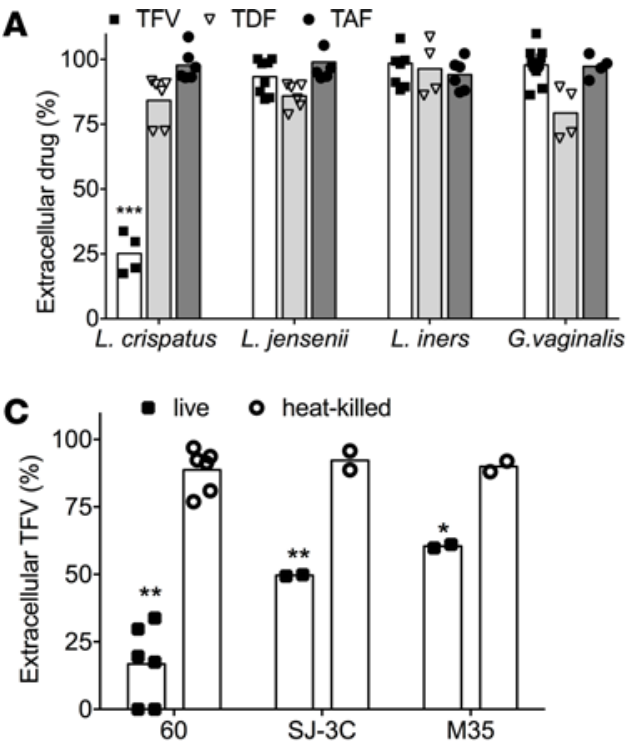

E
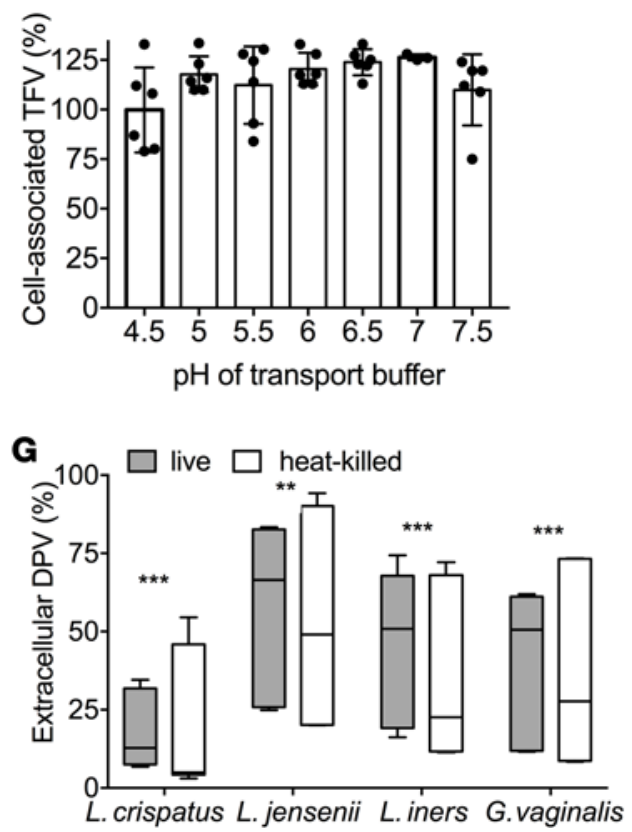

B

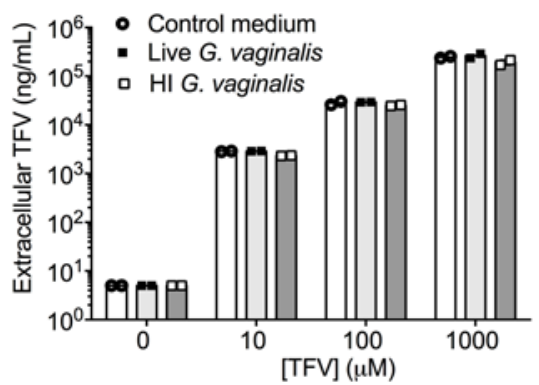

D

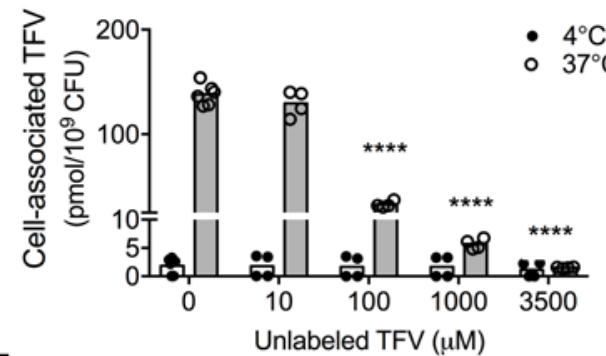

$\mathbf{F}$

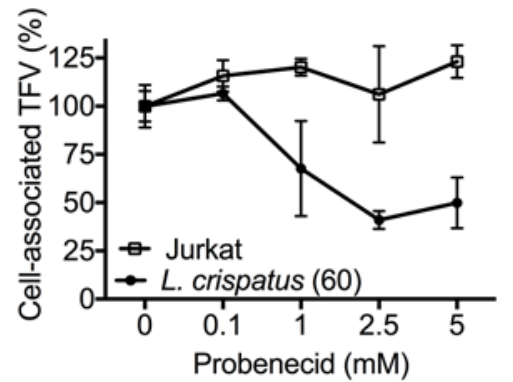

H

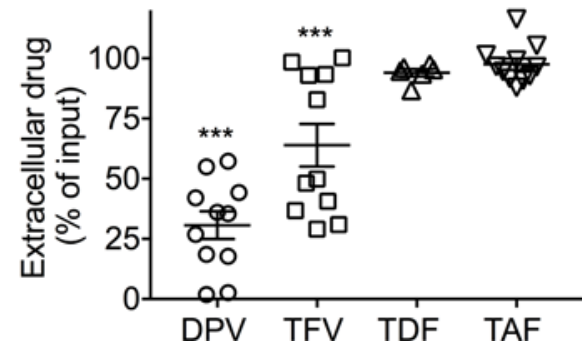

Figure 2. TFV and DPV pharmacokinetics are modified by vaginal microbiota. (A) Vaginal bacteria were exposed to $1 \mu \mathrm{M}$ radiolabeled TFV, TDF, or TAF for 2 hours at $37^{\circ} \mathrm{C}$, and radiolabeled drug recovered in the supernatants (extracellular drug) was quantified by liquid scintillation counting after pelleting the bacteria and expressed as percentage of input. (B) Tenofovir $(0,10,100$, or 1,000 $\mu \mathrm{M})$ was incubated with live or heat-inactivated G. vaginalis (strain 594) or NYC III medium without bacteria (control) for 6 hours at $37^{\circ} \mathrm{C}$ and the extracellular levels of drug were quantified by HPLC-MS/MS. Results are presented as mean \pm SEM of 2 independent experiments per condition. (C) Radiolabeled drug recovered in supernatant was quantified after incubating TFV with different species of live or heat-killed L. crispatus; (D) after incubation with $L$. crispatus (strain 60) at $4^{\circ} \mathrm{C}$ or $37^{\circ} \mathrm{C}$ in the presence of increasing concentrations of unlabeled drug; $(\mathbf{E})$ at different $\mathrm{pH}$; and $(\mathbf{F})$ in the presence of probenecid (0-5 mM). (C) Live or heat-killed bacteria were exposed to $1 \mu \mathrm{M}$ radiolabeled DPV and extracellular drug recovered quantified by scintillation counting as in panel $\mathbf{A}$. Results represent mean \pm SEM of 2 to 4 independent experiments, with each sample tested in duplicate. (H) Fresh vaginal swab eluents (without adjustment of $\mathrm{pH})$ were incubated with $\left.1 \mu \mathrm{M}\left[{ }^{3} \mathrm{H}\right] \operatorname{TFV}(n=11),{ }^{3} \mathrm{H}\right] \operatorname{TDF}(n=5),\left[{ }^{14} \mathrm{C}\right] \operatorname{TAF}(n=11)$, or $\left[{ }^{3} \mathrm{H}\right] \mathrm{DPV}(n=11)$ for 2 hours at $37^{\circ} \mathrm{C}$ and then subjected to centrifugation and filtration. The radioactivity recovered in the supernatants was quantified by scintillation counting and results are expressed as percentage of input radioactivity (mean and interquartile range). The asterisks indicate statistical significance by unpaired Student's $t$ test comparing extracellular drug recovery relative to abiotic controls (panels $\mathbf{A}, \mathbf{B}$, and $\mathbf{F}$ ) or by 1-way ANOVA with a Bonferroni's post hoc analysis compared to no unlabeled TFV (C), no probenecid (E), or TAF (C). ${ }^{*} P<0.05 ;{ }^{* *} P<0.01,{ }^{* * *} P<0.001 ;{ }^{* * *} P<0.0001$. 
Table 1. Bacterial gene copies and drug recovered in culture supernatants following incubation of radiolabeled drug with vaginal swabs

\begin{tabular}{|c|c|c|c|c|c|c|}
\hline Sample & pH & L. crispatus ${ }^{\mathrm{A}}$ & L. jensenii ${ }^{\mathrm{A}}$ & G. vaginalis ${ }^{\mathrm{A}}$ & TFV $^{B}$ & DPV $^{B}$ \\
\hline 1 & 4.4 & $2,928,624$ & 14,056 & 2.5 & 40.6 & 44.3 \\
\hline 2 & 4.6 & 2,100 & 32,119 & 2,047 & 82.8 & 35.4 \\
\hline 4 & - & 59,269 & 2.5 & 53.6 & 93.4 & 18.6 \\
\hline 5 & 4.4 & 2.5 & 2.5 & $2,052,876$ & 48.1 & 42.1 \\
\hline 6 & 4.2 & 2.5 & 11,833 & 2.5 & 29.0 & 36 \\
\hline 9 & 4.6 & 2.5 & 2.5 & $3,357,376$ & 49.9 & 55.0 \\
\hline 10 & 6.0 & 2.5 & 2.5 & 3,273 & 100.3 & 2.6 \\
\hline 11 & 5.5 & $3,749,730$ & 48,306 & 1,256 & 30.9 & 17.8 \\
\hline
\end{tabular}

${ }^{A}$ Bacterial copies were determined by quantitative PCR and extrapolated from a standard curve and values below the lower limit of detection were assigned half the lower limit. BDrug bioavailability was quantified as percentage of radiolabeled drug recovered in culture supernatants after incubating drug with swab eluents followed by centrifugation.

$\operatorname{TFV}(0,10,100$, or $1,000 \mu \mathrm{M})$ and cell-associated or extracellular drug levels measured by mass spectrometry (MS). Increasing concentrations of TFV were detected in L. crispatus (but not G. vaginalis) lysates and conversely, similar quantities of unlabeled TFV were recovered from supernatants following incubation with live or heat-inactivated $G$. vaginalis compared with abiotic controls (Figure $2 \mathrm{~B}$ ).

To explore the mechanism by which $L$. crispatus affected TFV recovery, additional studies were performed. TFV associated minimally with L. crispatus at $4^{\circ} \mathrm{C}$ and was susceptible to competition by excess unlabeled drug (Figure 2D). Moreover, TFV accumulation in L. crispatus was $\mathrm{pH}$ independent (Figure 2E), and significantly reduced in the presence of noncytotoxic concentrations of probenecid, an inhibitor of OAT1 and OAT3 (Figure 2F and Supplemental Figure 3A). In contrast, probenecid did not inhibit TFV uptake by Jurkat cells, which do not express these OATs (10). Additionally, TFV was metabolized rapidly into TFV-DP by L. crispatus (Supplemental Figure 2). Together, these findings suggest active transport and metabolism of TFV by L. crispatus.

DPV bound nonspecifically to plastic plates and therefore, we modified the experimental approach and compared radiolabeled drug recovery in supernatants in the absence or presence of bacteria when DPV was added to polypropylene tubes containing $5 \mathrm{mg} / \mathrm{ml} \mathrm{BSA}$ as a blocking agent. There was a significant reduction in radioactivity recovered in the presence of live or heat-killed bacteria relative to radiolabeled drug detected when drug was added to abiotic tubes (culture medium only) (Figure 2G).

Incubation of TFV or DPV with vaginal swabs from women results in decrease in drug bioavailability. To test whether the observed effects of individually cultured bacteria on drug PK translate to what might happen clinically when drugs are exposed to multiple bacterial species of variable density and at different stages of growth, radiolabeled drugs were exposed to fresh vaginal swab eluates for 2 hours and the drug remaining in supernatants was quantified after centrifugation. There was a $69.3 \% \pm 6 \%$ and a $36.1 \% \pm 9 \%$ mean decrease in extracellular DPV and TFV, respectively, but no loss of TAF or TDF (Figure $2 \mathrm{H})$. The bacterial copies/swab of L. crispatus, L. jensenii, or G. vaginalis were determined by quantitative PCR (qPCR) and there was no significant correlation between bacterial copies of L. crispatus, L. jensenii, or G. vaginalis and the percentage of extracellular drug recovered $(P>0.05$, Spearman's correlation) (Table 1$)$. Notably, the 2 samples with greater than $10^{6}$ L. crispatus copies/swab, a concentration of live L. crispatus that reduced the percentage of extracellular TFV recovered when radiolabeled drug was incubated with bacteria in vitro, resulted in a substantial reduction in extracellular TFV recovered. However, other samples with little L. crispatus also reduced TFV recovery. These findings suggest that additional bacterial species or their products may adversely impact TFV drug bioavailability (Table 1).

Decrease in radiolabeled drug detected in culture supernatants translates to a reduction in HIV inhibitory activity. To determine if the decrease in drug availability following exposure to different bacteria translated to a shift in the concentration of drug needed to inhibit HIV infection, live or heat-killed L. crispatus (strain 60) or G. vaginalis (strain 594) were exposed to increasing concentrations of drug, bacteria pelleted, and the culture 
A

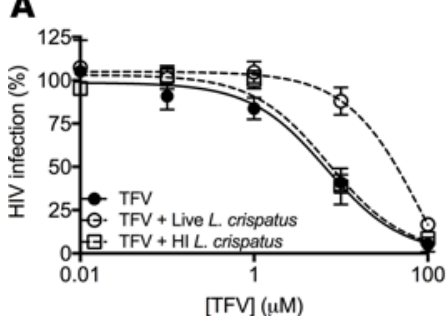

[TFV] $(\mu \mathrm{M})$
B

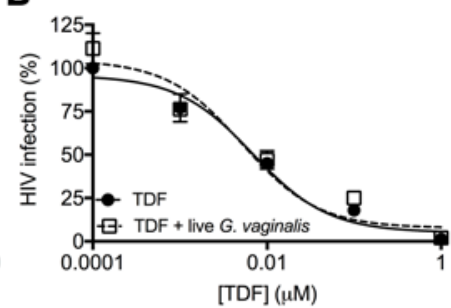

C

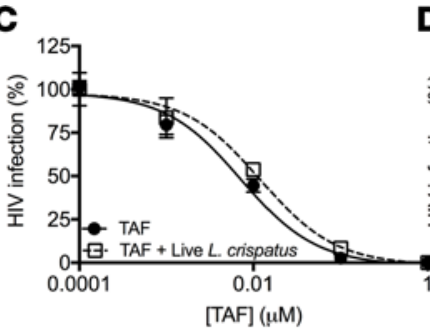

D

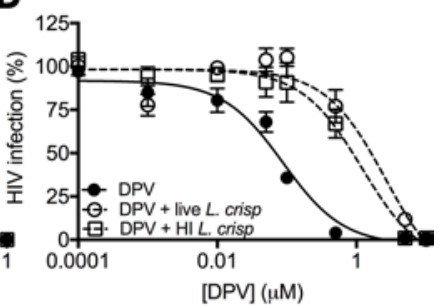

E

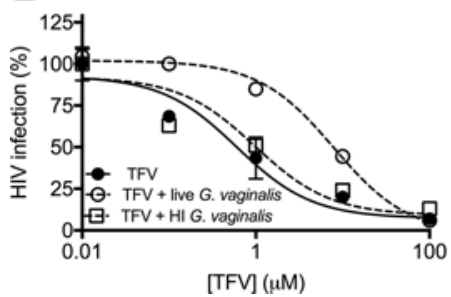

F

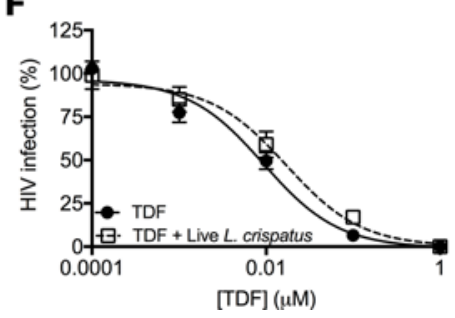

G

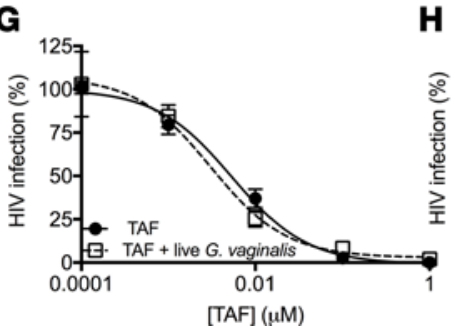

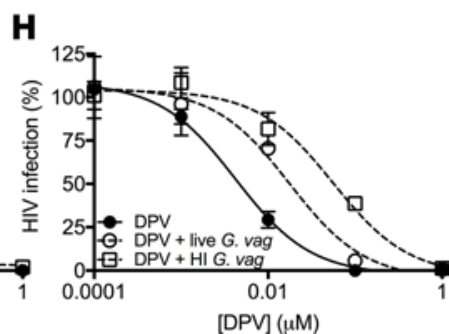

Figure 3. HIV inhibitory activity of drug is impacted following incubation with vaginal microbiota. Increasing concentrations of unlabeled drug were incubated with live or heat-inactivated (HI) L. crispatus (strain 60) or G. vaginalis (strain 594) in PBS with 25 mM glucose or with buffer alone (abiotic controls) for 2 hours at $37^{\circ} \mathrm{C}$ and then subjected to centrifugation and filtration. The $\mathrm{pH}$ of the culture supernatants was adjusted to 7.3 and then assayed for anti-HIV activity in TZM-bl assays. Results are representative of 3 independent experiments each conducted in duplicate. The IC ${ }_{50}$ concentrations of the drugs were interpolated using nonlinear regression analysis.

supernatants tested for anti-HIV activity. The concentration of drug needed to inhibit $50 \%$ of $\mathrm{HIV}$ infection $\left(\mathrm{IC}_{50}\right.$ ) increased 13-fold from $6.7 \mu \mathrm{M}$ to $89.6 \mu \mathrm{M}$ (Figure 3A) when TFV was preincubated with live (but not heat-killed) L. crispatus compared with abiotic cultures. TDF and TAF had little effect on the $\mathrm{IC}_{50}$ (Figure $3, \mathrm{~B}$ and $\mathrm{C})$. As expected, the $\mathrm{IC}_{50}$ for DPV increased when drug was preincubated with live or heat-killed L. crispatus or G. vaginalis (Figure 3, D and $\mathrm{H}$ ). However, the $\mathrm{IC}_{50}$ for TFV, but not TDF or TAF, also increased approximately 13.1-fold when drug was preincubated with live, but not heat-killed, G. vaginalis (Figure 3, E-G). This result was unexpected, as there was no reduction in drug recovered either by quantifying radioactivity or by MS in the culture supernatants when TFV was preincubated with $G$. vaginalis, and suggested that a bacterial metabolite or product secreted into the culture supernatants might interfere with TFV antiviral activity by other mechanisms.

Modulation of drug uptake by bacterial products. To test the hypothesis that $G$. vaginalis or other bacteria secrete metabolites that impact drug PK or pharmacodynamics (PD), radiolabeled TFV or TDF $(0.5 \mu \mathrm{M})$ was added to Jurkat $\mathrm{T}$ cells in the presence of bacterial culture supernatants or control broth (normalized to $\mathrm{pH}$ 6.8). After a 1-hour incubation period, the cells were washed extensively and Jurkat cell-associated radioactivity quantified. Relative to control broth, culture supernatants (1:4 dilution) isolated from $L$. crispatus, L. jensenii, L. iners, 2 strains of G. vaginalis (DNF01662 and KA00735), Prevotella bivia, and Mageeibacillus indolicus (BVAB3) significantly reduced uptake of $\left[{ }^{3} \mathrm{H}\right] \mathrm{TFV}$, whereas Atopobium vaginae culture supernatants significantly increased $\left[{ }^{3} \mathrm{H}\right] \mathrm{TFV}$ uptake (Figure 4A). None of the supernatants had any significant effects on TDF. To evaluate the potency of this effect, additional studies were conducted with increasing dilutions of culture supernatants and with a higher concentration of TFV $(50 \mu \mathrm{M})$. G. vaginalis strain DNF01662, $L$. crispatus, L. iners, and P. bivia lost their activity at a dilution of 1:64, whereas G. vaginalis (KA00735) and $A$. vaginae (DNF00720) lost activity at dilutions of 1:16 and 1:32, respectively (Figure 4B).

$A$. vaginae and $G$. vaginalis, which belong to the same phylum (Actinobacteria), frequently coexist and are highly prevalent in the setting of BV (23), but induced opposing effects on TFV uptake. Hence, we focused on the more potent strains of these species for subsequent mechanistic studies (1:32 dilution of $G$. vaginalis DNF01662 and 1:8 dilution of $A$. vaginae DNF00720). The effects were not cell type specific, as $G$. vaginalis and $A$. vaginae culture supernatants inhibited or enhanced TFV uptake by Jurkat cells, primary CD4 ${ }^{+} \mathrm{T}$ cells, TZM-bl cells, VK2E6/E7 cells, and human keratinocytes (HaCAT) (Figure 4C). Moreover, the supernatants had the same opposing effects on a structurally similar drug, adefovir (ADF), which is also transported into human cells by endocytosis, but had no impact on other passively transported drugs, such as ADF dipivoxil 
A

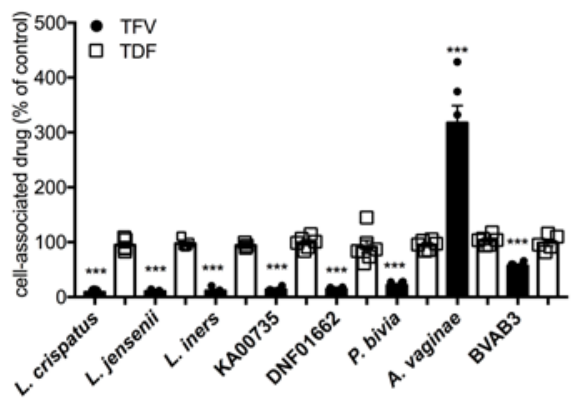

D

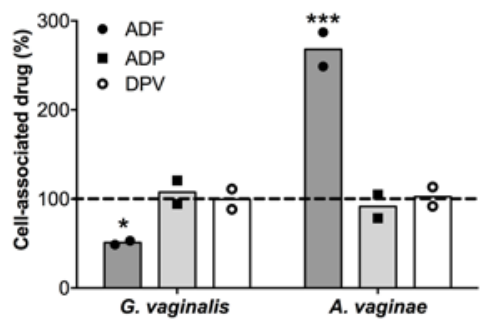

B

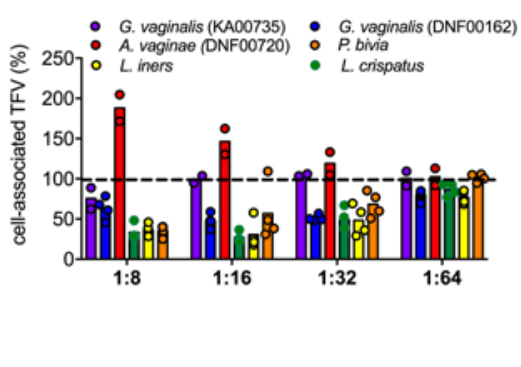

E

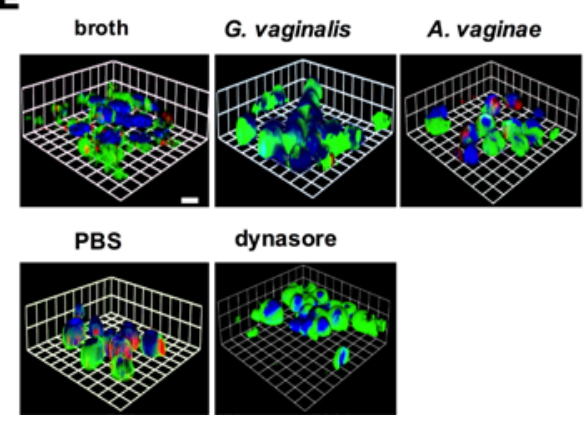

C

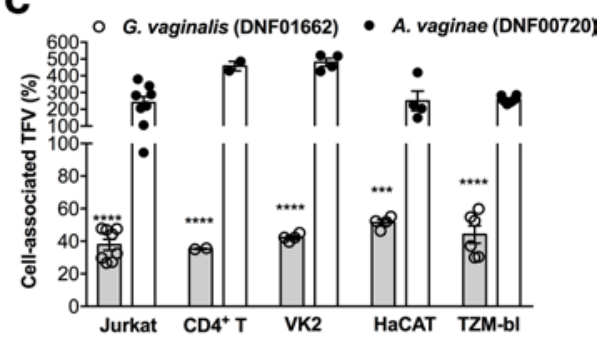

$\mathbf{F}$

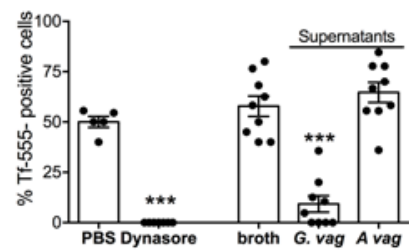

Figure 4. Effects of bacterial supernatants on drug uptake. (A) Bacterial culture supernatants (1:4 dilution) were applied to Jurkat cells for 1 hour prior to the addition of $0.5 \mu \mathrm{M}\left[{ }^{3} \mathrm{H}\right] \mathrm{TFV}$ or $\left[{ }^{3} \mathrm{H}\right] \mathrm{TDF}$ and Jurkat cell-associated drug quantified by liquid scintillation counting. (B) Supernatants from indicated strains of $G$. vaginalis, A. vaginae, L. crispatus, L. iners, or P. bivia were diluted 1:8 to 1:64 in PBS and applied to cells for 1 hour prior to addition of $50 \mu \mathrm{M}\left[{ }^{14} \mathrm{C}\right] \mathrm{TFV}$. Data are presented as mean \pm SEM from at least 2 independent experiments with duplicate measurements per condition. (C) Different human cell lines or primary CD4 ${ }^{+} T$ cells were exposed to 1:8 dilution of $A$. vaginae (DNF00720) or 1:32 dilution of $G$. vaginalis (DNF00162) supernatants for 1 hour prior to exposure to $0.5 \mu \mathrm{M}$ radiolabeled drugs. Results are mean \pm SEM of duplicate measurements from at least 2 independent experiments. (D) Bacterial culture supernatants (1:4 dilution) were applied to Jurkat cells for 1 hour prior to the addition of radiolabeled adefovir (ADF), adefovir dipivoxil (ADP), or dapivirine DPV and cell-associated drug quantified by scintillation counting. Results are mean \pm SEM of duplicate measurements from 2 independent experiments. (E) Jurkat cells were incubated with the indicated culture supernatants, abiotic control broth, 80 $\mu \mathrm{M}$ dynasore, or PBS prior to exposure to $50 \mu \mathrm{g} / \mathrm{ml}$ of Alexa Fluor 555-conjugated transferrin (Tf-555) in the presence of the supernatants. The plasma membranes were stained green with DiO, nuclei blue with DAPI, and the transferrin is red. Representative images from 2 independent experiments are shown. (F) Approximately 100 cells from 6-9 randomly selected fields were counted and the percentage of cells that internalized Tf-555 is shown. The asterisks indicate significant differences relative to abiotic controls by unpaired Student's $t$ test (A and F) or by ANOVA with Tukey's multiple-comparison correction (C and $\mathbf{D}) .{ }^{*} P<0.05 ;{ }^{* *} P<0.001 ;{ }^{* * *} P<0.0001$.

(ADP) or DPV (Figure 4D) (10, 24). G. vaginalis supernatants also inhibited endocytosis of transferrin (Figure $4, \mathrm{E}$ and $\mathrm{F})$. Dynasore $(80 \mu \mathrm{M})$ was included as a positive control for inhibition of endocytosis. A. vaginae culture supernatants elicited a small but not statistically significant increase in transferrin internalization.

Adenine mediates the inhibitory activity of $G$. vaginalis supernatants. The inhibitory activity of the culture supernatants was heat stable, not affected by digestion with proteolytic enzymes, and mapped to a $<0.5-\mathrm{kDa}$ fraction after centrifugation by a $3-\mathrm{kDa}$ pore-size Amicon filter and subsequent dialysis across a 0.1- to 0.5$\mathrm{kDa}$ membrane. The dialysate was further fractionated by high-performance liquid chromatography (HPLC) and the UV chromatogram of the eluted fractions demonstrated a pronounced peak at approximately 12-13 minutes (Figure 5A). This peak exhibited maximum absorbance at $260 \mathrm{~nm}$, consistent with the properties of nucleotides and nucleotide-like substances. Fraction 4, which corresponded to the peak, had the most potent inhibitory activity (Figure 5B). MS analysis of this fraction and comparison of the retention time and $\mathrm{m} / \mathrm{z}$ ratio with those of an adenine standard suggested the molecule was adenine. Moreover, cell culture-grade adenine significantly decreased $\left[{ }^{3} \mathrm{H}\right] \mathrm{TFV}$ uptake relative to the diluent (PBS) at concentrations greater than $10 \mu \mathrm{M}$ (Figure 5). Adenine was detected at $246 \mu \mathrm{M}$ in $G$. vaginalis supernatants compared with $65.5 \mu \mathrm{M}$ in PYG-YG broth and only at $9.5 \mu \mathrm{M}$ in $A$. vaginae supernatants. Concentrations of adenine in L. crispatus supernatants were similarly high $(\sim 281 \mu \mathrm{M})$, consistent with its inhibitory activity (Figure $4 \mathrm{~A})$.

Inhibition of TFV endocytosis by $G$. vaginalis culture supernatants or adenine translates to an increase in the $I C_{50}$. TZM-bl cells were preincubated with increasing doses of TFV, TDF, or TAF in the absence or presence of $G$. vaginalis culture supernatants (diluted $1: 2,1: 4$, or $1: 8$ ) or adenine $(10,100$, or $1,000 \mu \mathrm{M}$ ). No cytotoxicity was 
A

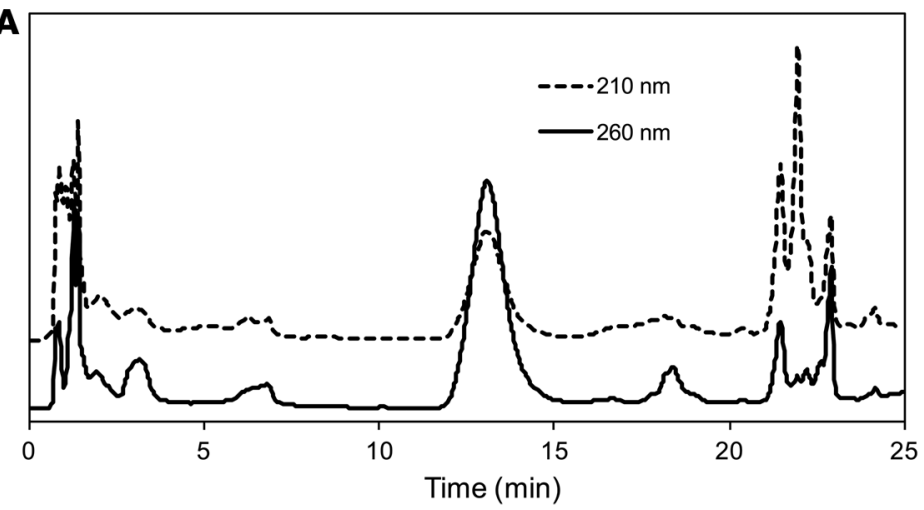

B

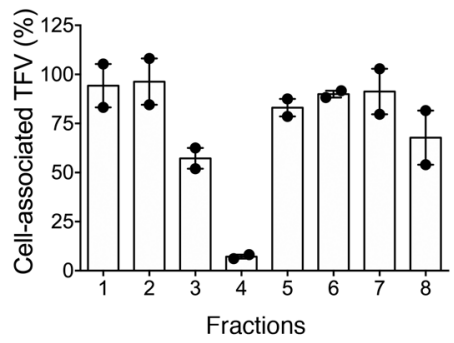

C

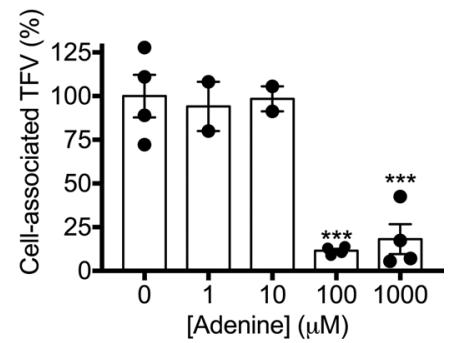

Figure 5. Adenine mediates the inhibitory activity of

G. vaginalis supernatants. (A) The bioactive dialysate $(<0.5$ kDa) was fractionated by HPLC. (B) Fractions collected by HPLC were applied to Jurkat cells for 1 hour prior to addition of 0.5 $\mu \mathrm{M}\left[{ }^{3} \mathrm{H}\right] \mathrm{TFV}$. (C) Jurkat cells were exposed to adenine $(0-1,000$ $\mu \mathrm{M})$ for 1 hour prior to addition of $0.5 \mu \mathrm{M}\left[{ }^{3} \mathrm{H}\right] \mathrm{TFV}$. For panels $\mathbf{B}$ and $\mathbf{C}$, cell-associated radioactivity was quantified using liquid scintillation counting. Data represent mean \pm SEM from 2 independent experiments with duplicate conditions per sample. ${ }^{* * *} P<0.001$ by 1 -way ANOVA with Bonferroni's post hoc test.

observed when TZM-bl cells were incubated with these concentrations of G. vaginalis culture supernatants or adenine for 48 hours (Supplemental Figure 3, B and C). The addition of G. vaginalis culture supernatants or adenine resulted in a dose-dependent increase in the $\mathrm{IC}_{50}$ for TFV. Specifically, the TFV $\mathrm{IC}_{50}$ increased 19-fold from 0.95 to $18.0 \mu \mathrm{M}$ in the presence of $G$. vaginalis (1:2 dilution) compared with abiotic culture supernatants (Figure 6A) and 18.2-fold from 0.6 to $11.2 \mu \mathrm{M}$ in the presence of $100 \mu \mathrm{M}$ adenine (Figure $6 \mathrm{D}$ ). In contrast, at the highest concentrations, neither the $G$. vaginalis culture supernatants nor adenine had any impact on the TDF or TAF dose-response curves (Figure 6, B, C, E, and F).

\section{Discussion}

While the effects of the gut microbiome on the PK of oral drugs have been well studied (25), the potential for the vaginal microbiome to modulate drug PK has received much less attention. A link between vaginal microbiome and TFV PK was first suggested in a subanalysis of CAPRISA 004 samples in which the efficacy of TFV gel was less in women with a non-lactobacillus-dominant microbiome (19). The authors suggested that $G$. vaginalis might metabolize TFV. The current studies further support the role of the vaginal microbiome in modulating drug PK and antiviral activity and identify several diverse and complex mechanisms. These include binding and/or intracellular accumulation and metabolism of drugs directly by bacteria, inhibition of drug uptake by human cells in response to soluble bacterial products, or modulation of drug transport in response to changes in vaginal $\mathrm{pH}$. All 3 of the mechanisms affected TFV drug levels resulting in a shift in the $\mathrm{IC}_{50}$ against $\mathrm{HIV}$, while DPV was only impacted by nonspecific binding to bacterial species. There was little impact of bacteria on bioavailability of drug and the HIV inhibitory dose-response curves for the prodrugs, TDF and TAF.

First, we observed a decrease in TFV transport into human cells as $\mathrm{pH}$ increased to levels observed following exposure to semen or in the setting of BV. The impact of $\mathrm{pH}$ on TFV uptake by human cells is consistent with an endocytic transport mechanism (26), and may have contributed to the decreased efficacy of TFV observed in CAPRISA 004 comparing women with a lactobacillus-dominant versus non-lactobacillus-dominant microbiome (19). In contrast, TDF uptake was greater at $\mathrm{pH}>6$ compared with $\mathrm{pH} 4.5$, which could reflect inhibition of the enzymes that are involved in hydrolysis of TDF to TFV at the low $\mathrm{pH}$ resulting in TDF diffusing out of the cells.

Second, and surprisingly, we found that TFV is actively transported into and metabolized by L. crispatus, which resulted in less drug being available for uptake by human cells and translated to an increase in the in vitro 
A

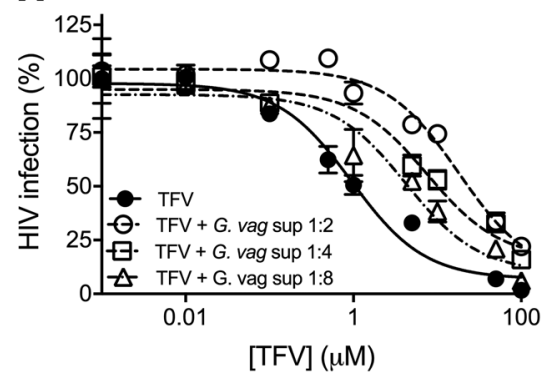

D

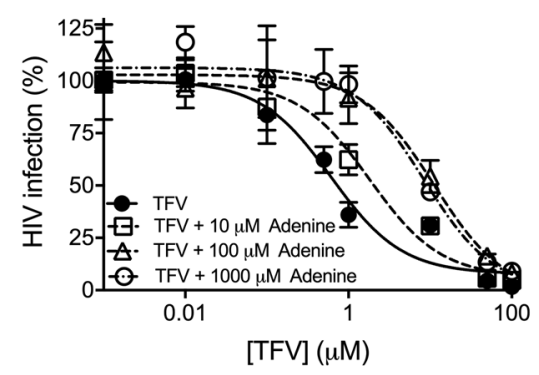

B

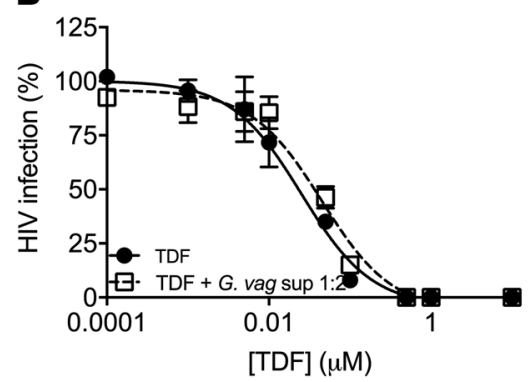

E

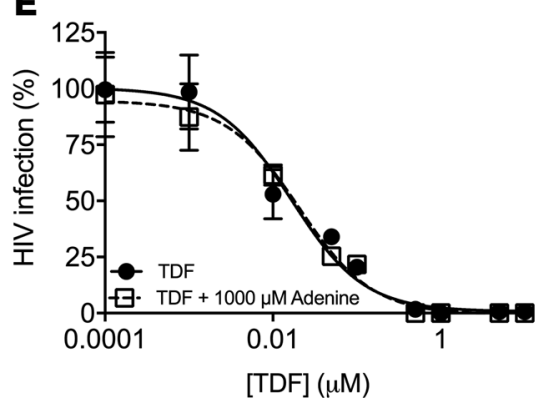

C

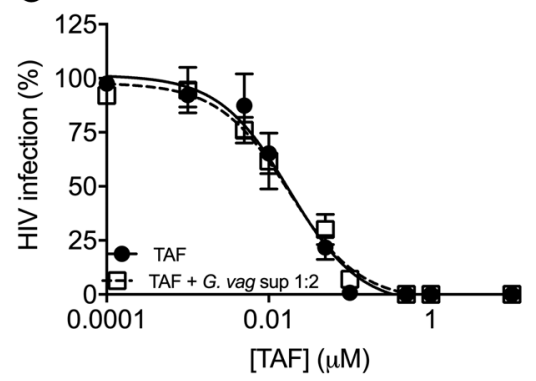

F

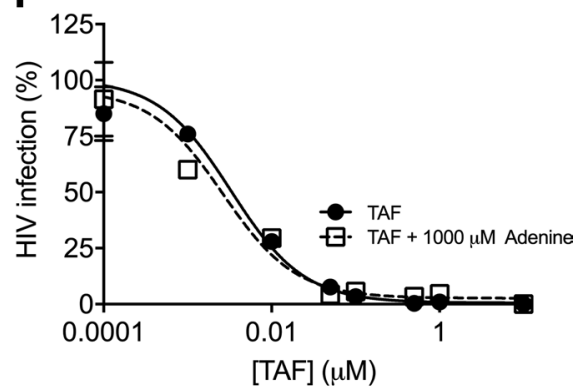

Figure 6. G. vaginalis culture supernatants or adenine reduce the antiviral activity of TFV, but not TDF or TAF. Drugs were incubated with control buffer or with the indicated dilutions of $G$. vaginalis culture supernatants $(\mathbf{A}-\mathbf{C})$ or indicated concentrations of adenine (D-F) and then applied to TZM-bl cells for 2 hours prior to infection with HIV. Results are presented as the mean \pm SEM of a representative of 2-3 independent experiments. $\mathrm{IC}_{50}$ concentrations of the drugs were interpolated using nonlinear regression analysis.

$\mathrm{IC}_{50}$. Unlike human $\mathrm{T}$ cells, the transport of TFV by L. crispatus was $\mathrm{pH}$ independent, but blocked by probenecid, suggesting an OAT-mediated mechanism (27). Genomic databases indicate that lactobacilli may express several anionic transporters and drug-metabolizing enzymes, but further studies are needed to define the specific ones involved in TFV uptake and metabolism by L. crispatus (27). While this observation suggests that $L$. crispatus may act as sink, trapping TFV as TFV-DP for several days, it could subsequently serve as a reservoir, releasing TFV into the vaginal mucosa after the drug is dephosphorylated back to TFV and transported out of bacterial cells. Notably, efficiency of TFV transport varied between strains of $L$. crispatus and neither $L$. jensenii nor $L$. iners accumulated TFV, suggesting strain- and species-specific differences in transporter expression.

Although it was previously suggested that $G$. vaginalis metabolizes TFV to adenine (19), we did not detect any direct intracellular accumulation of TFV by different strains of $G$. vaginalis even after more prolonged incubation either using radiolabeled drug or MS. The differences between the previous and current study could reflect differences in in vitro growth conditions. Instead, studies with bacterial culture supernatants uncovered a third mechanism by which $G$. vaginalis and other bacterial species may modulate PK. $G$. vaginalis released adenine into culture supernatants, which blocked TFV (and ADF and transferrin) transport into human cells by inhibiting endocytosis. Interestingly, $G$. vaginalis secreted, whereas A. vaginae consumed, adenine. This suggests a previously unrecognized economic interaction between 2 bacterial species that frequently coexist (23). Secretion of adenine may be common to other vaginal bacteria, as increases in adenine levels and a concomitant inhibition of TFV uptake were observed with culture supernatants from other species. Clinical studies quantifying adenine and other metabolites present in cervicovaginal secretions, their link to differences in microbial community states, and the impact of microbiome on TFV drug PK are needed to better understand the implications of these findings.

The complex mechanisms by which bacteria may modulate TFV PK may have contributed to the lack of statistically significant correlation between bacterial copy numbers and recovery of radiolabeled TFV. Notably, only 2 of the participants had more than $10^{6}$ copies/swab of L. crispatus and both of those had low levels of TFV recovered (40.6\% and 30.9\%). However, a low percentage of TFV was also recovered after incubating the drug with other swabs that had little or no L. crispatus detected. This suggests that other bacterial species or molecules in the swab eluates that were not quantified reduce drug availability. Larger, 
more comprehensive studies are needed to elucidate the multiple mechanisms by which vaginal microbiota or their products modulate TFV PK.

The inhibitory effects of microbiota and/or soluble products on TFV or DPV PK were overcome at higher drug levels, as illustrated by the HIV infection dose-response curves and, thus, would be predicted to have little effect in the setting of sustained drug delivery and high levels of adherence. However, as drug levels decrease between doses or with intermittent adherence, the microbiome could contribute to a further reduction in protection for TFV or DPV. For example, if a DPV ring were removed before sex, the drug would rapidly transit out of human cells along a concentration gradient, and could then bind irreversibly to bacteria and semen (29), resulting in loss of protection. A phase 1 DPV IVR PK study documented rapid decay in cervical tissue and cervicovaginal fluid drug levels, suggesting a short window of time between ring removal and loss of HIV prevention (28).

In contrast, because of the prolonged intracellular half-life of TFV-DP, TFV-based PrEP would be predicted to provide a longer window of protection following ring removal or missed doses of gel or film formulations compared with DPV. However, there may not be sufficient extracellular TFV available during periods of ring removal or missed doses to overcome the inhibitory effects of $\mathrm{pH}$ or adenine on TFV endocytosis and to protect immune cells recruited into the FGT in response, for example, to inflammatory stimuli. This notion is supported by the finding that TFV concentrations in cervicovaginal fluid and tissue varied significantly among study participants, particularly following barrier-unprotected sex (30). The finding that some strains of L. crispatus (and possibly other bacterial species) compete with and may be more efficient at transporting and metabolizing TFV compared with $\mathrm{T}$ cells increases the complexity of the interactions between TFV and the vaginal microbiome.

None of the mechanisms studied (except for an increase in TDF transport at higher $\mathrm{pH}$ ) impacted the PK or HIV inhibitory dose curves for TDF or TAF. These findings, coupled with the ability of these prodrugs to achieve higher intracellular levels because they passively diffuse into cells rather than relying on endocytosis or active transport, suggest that TDF or TAF may provide more consistent and higher intracellular TFV-DP levels than TFV and could be more forgiving than TFV or DPV.

Identifying whether the vaginal microbiome and other mucosal environmental factors modulate antiretroviral $\mathrm{PK}$ and determining the underlying mechanisms provides insights into which drugs, delivery systems, and dosing strategies are optimal for topical HIV prevention. The reduced efficiency of TFV endocytosis at non-acidic $\mathrm{pH}$ and its inhibition by soluble bacterial products, such as adenine, may have contributed to the differential efficacy observed in clinical trials. Maintaining an acidic $\mathrm{pH}$ environment and promoting a lactobacillus-dominant microbiome could enhance TFV uptake. However, the ability of some strains of L. crispatus to actively transport and metabolize TFV complicates drug PK. DPV is also impacted by the mucosal environment because it binds irreversibly and nonselectively to bacteria, epithelial cells, and semen resulting in an increase in the $\mathrm{IC}_{50}$ required to inhibit HIV infection in vitro (29). This characteristic may contribute to a requirement for a high level of consistent adherence reflected in the clinical trial outcomes. Together with clinical trial experiences, these studies highlight the importance of evaluating the impact of the microbiome and other mucosal factors on drug PK and efficacy. Selection of drugs that are less susceptible to environmental factors such as the prodrugs, TDF and TAF, may lead to more consistent efficacy in the realistic setting of imperfect adherence.

\section{Methods}

Reagents. Unlabeled and $\left[{ }^{14} \mathrm{C}\right]$-labeled TAF $(57 \mathrm{mCi} / \mathrm{mmol})$, unlabeled $\mathrm{TDF}$, and unlabeled TFV were provided by Gilead Sciences, Inc. DPV was purchased from MedKoo Biosciences, Inc. [ $\left.{ }^{3} \mathrm{H}\right] \mathrm{TDF}$ (1.3-5.6 Ci/ mmol), $\left[{ }^{3} \mathrm{H}\right]$ TFV (8.1-17.1 Ci/mmol), $\left[{ }^{3} \mathrm{H}\right] \mathrm{DPV}$ (10 Ci/mmol), $\left[{ }^{14} \mathrm{C}\right] \mathrm{DPV}(59 \mathrm{mCi} / \mathrm{mmol}),\left[{ }^{3} \mathrm{H}\right]$ adefovir $(11.9 \mathrm{Ci} / \mathrm{mmol})$, and $\left[{ }^{3} \mathrm{H}\right]$ adefovir dipivoxil $(11.4 \mathrm{Ci} / \mathrm{mmol})$ were obtained from Moravek Biochemicals.

Cells, viruses, and bacteria. Immortalized human vaginal epithelial cells (VK2/E6E7 and CRL2616), TZM-bl, Jurkat, and HaCAT cells were obtained from the American Type Culture Collection (ATCC) and cultured as described $(10,12)$. HIV- $1_{\text {BaL }}$ were acquired through the NIH AIDS Reagent Program, Division of AIDS, National Institute of Allergy and Infectious Diseases (NIAID), NIH, and grown as described $(12,31)$. The bacterial strains used and culture conditions are detailed in Table 2. For drug uptake experiments, the bacterial cells were grown under microaerophilic conditions $(5 \%$ $\mathrm{CO}_{2}$ ) to exponential phase, and normalized to the same density for different strains before radiolabeled drugs were added. The turbidity of 18-hour cultures was measured using an Ultrospec 2100 Pro 
Table 2. Source and growth conditions of bacterial strains

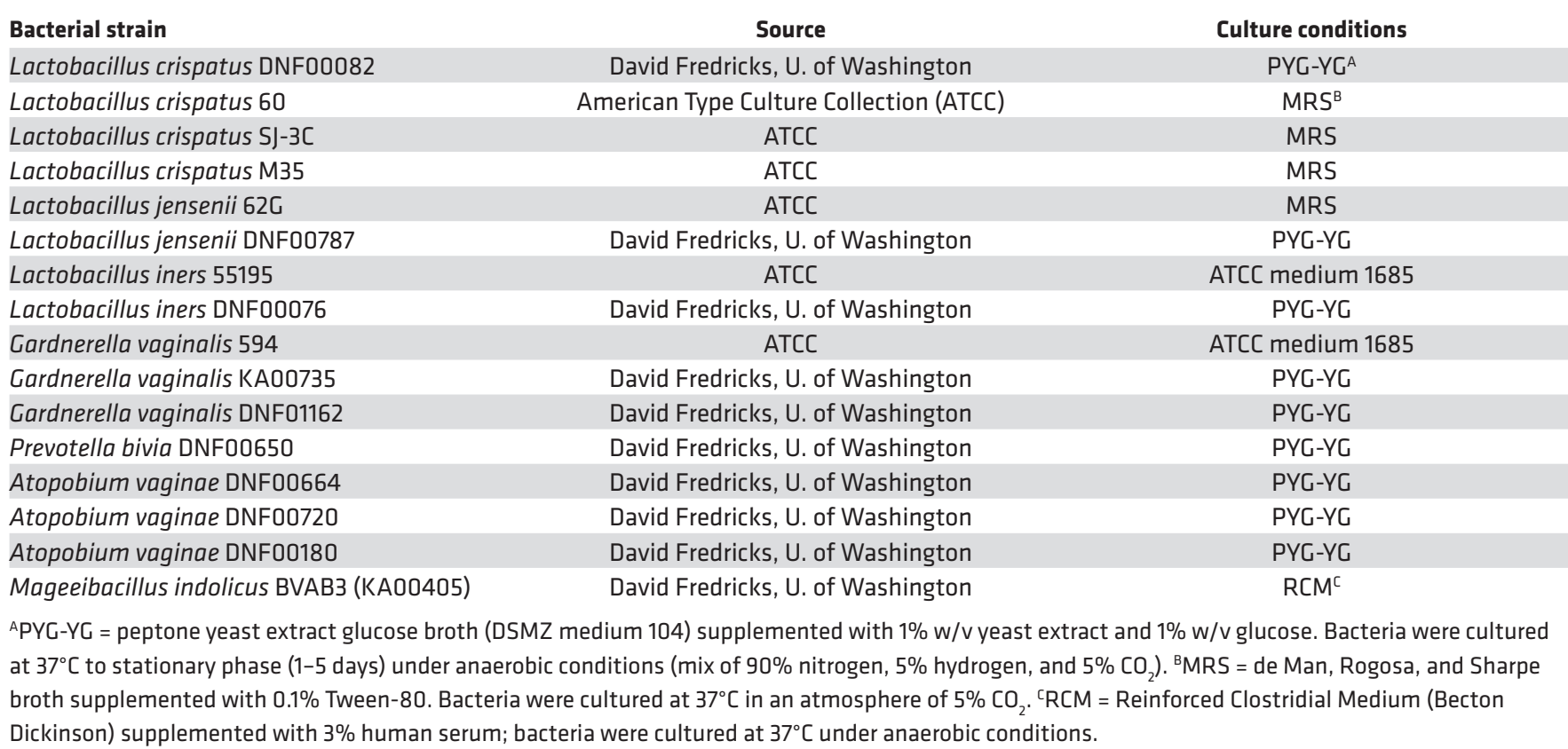

spectrophotometer (Amersham Biosciences), assuming that an optical density at $600 \mathrm{~nm}\left(\mathrm{OD}_{600}\right)$ of 1 corresponds to $0.25 \times 10^{9} \mathrm{CFU} / \mathrm{ml}$ for lactobacilli (32) and $0.5 \times 10^{9} \mathrm{CFU} / \mathrm{ml}$ for G. vaginalis (33). Bacterial culture supernatants were prepared by centrifugation of cultures at 3,000 rpm and filtration $(0.22-\mu \mathrm{m}$ syringe filter). Supernatants were generated from a single batch of bacteria and were aliquoted and stored frozen at $-80^{\circ} \mathrm{C}$. Four different batches of supernatants were used and recovered under the same conditions.

Radiolabeled drug transport into human or bacterial cells. Radiolabeled drug uptake in the absence or presence of bacterial culture supernatants, adenine, or $\mathrm{pH}$ was monitored as previously described (10). For $\mathrm{pH}$ studies, the cells were exposed to radiolabeled drugs in transport medium $(137 \mathrm{mM} \mathrm{NaCl}, 5.8$ $\mathrm{mM} \mathrm{KCl}, 1.8 \mathrm{mM} \mathrm{CaCl}_{2}, 1.2 \mathrm{mM} \mathrm{MgSO}_{4}, 10 \mathrm{mM}$ HEPES or MES, and $11 \mathrm{mM}$ glucose) adjusted to the indicated $\mathrm{pH}$ with $\mathrm{NaOH}$ or $\mathrm{HCl}$. To quantify uptake by bacteria, bacterial suspensions were washed with minimal salt medium (M9) containing $0.4 \%$ glucose, and resuspended in the same medium to $\mathrm{OD}_{600}$ $=4.0$ and exposed to radiolabeled drug in 2-ml 96-deep-well plates (USA Scientific) containing 0.4-0.8 $\mathrm{ml}$ of bacterial suspension $\left(\sim 1 \times 10^{9} \mathrm{CFU} /\right.$ sample), heat-killed bacteria $\left(95^{\circ} \mathrm{C}\right.$ for 15 minutes), or $\mathrm{M} 9$ alone. Due to adherence of DPV to the plates, DPV studies were conducted in 1.5-ml polypropylene tubes with PBS containing $5 \mathrm{mg} / \mathrm{ml}$ BSA as a coating buffer. After a 2-hour incubation and centrifugation at 2,500 rpm for 5 minutes to remove bacterial cells, the extracellular radioactivity in supernatants was measured and expressed as percentage of drug recovered in the abiotic plates or tubes. Additionally, vaginal swabs were collected from 11 premenopausal women between 21 and 45 years of age. The swabs were resuspended in $500 \mu \mathrm{PBS}$ (with $25 \mathrm{mM}$ glucose) to elute bacteria and cells, and $100 \mu 1$ of fresh eluate from each swab was incubated with radiolabeled drug at $37^{\circ} \mathrm{C}$ for 2 hours. The $\mathrm{pH}$ of the vaginal swab eluates varied from 3.8 to 6.0 (Table 1), and was not adjusted prior to addition of radiolabeled drugs. The drug-eluate mixture was subjected to centrifugation and filtration and the radioactivity in the supernatants quantified by liquid scintillation counting.

Drug metabolism in human and bacterial cells. Jurkat cells $\left(10^{7}\right.$ cell/sample), L. crispatus 60 (109 CFU/ sample), or $G$. vaginalis were exposed to 10,100 , and 1,000 $\mu \mathrm{M}$ TFV for the indicated times. Drug uptake was terminated by washing the cells with ice-cold medium and at selected times, cells were lysed in $40 \%$ methanol $/ 40 \%$ acetonitrile. Drug levels in cellular lysates or in the extracellular medium were quantified by HPLC-MS (10). Cell-associated drug levels were normalized by approximated cell volumes $(34,35)$. 
Antiviral activity. TZM-bl cells were treated with antiviral drugs in the absence or presence of $G$. vaginalis culture supernatants isolated from fresh cultures $(1: 2,1: 4$, or 1:8 dilution), adenine $(1-1,000 \mu \mathrm{M})$, or after incubation with live or heat-killed $L$. crispatus or $G$. vaginalis. For studies with $L$. crispatus and $G$. vaginalis, drugs were mixed with freshly cultured bacteria $\left(\mathrm{OD}_{600}=4\right)$ resuspended in $\mathrm{PBS}$ supplemented with $25 \mathrm{mM}$ glucose, $5 \mathrm{mg} / \mathrm{ml} \mathrm{BSA}$, and $0.001 \%$ DMSO (control buffer) or with the control buffer for 2 hours at $37^{\circ} \mathrm{C}$. The bacteria-drug mixture was then subjected to centrifugation, and the resulting supernatants filtered $(0.22-\mu \mathrm{m}$ filter), and $\mathrm{pH}$ adjusted to 7.3 prior to adding it to the TZM-bl cells. For studies with $G$. vaginalis supernatants, the bacteria were grown to stationary phase and were then pelleted, washed, and cultured for an additional 6 hours in NYC III media diluted 1:8 with PBS. Culture supernatants were harvested by centrifugation of the bacterial cell pellet and further diluted as indicated in PBS, and added to TZM-bl cells in the presence of drugs for 2 hours. After exposure to the drug-supernatant mixture, TZM-bl cells were infected with HIV- $1_{\mathrm{BaL}}\left(2 \times 10^{3} 50 \%\right.$ tissue culture infective dose) for approximately $30-48$ hours, and infection quantified in relative luminescence units as a percentage of that in the absence of drugs.

Quantitative $16 S$ rRNA PCR. qPCR reactions for L. crispatus, L. jensenii, and G. vaginalis were performed using primers and a dual-labeled fluorogenic probe hydrolyzed during PCR specific for each bacterium's 16S rRNA gene as previously described (36).

Confocal microscopy. Jurkat cells $\left(5 \times 10^{6}\right.$ cells/sample) were pretreated with bacterial culture supernatant or dynasore $(80 \mu \mathrm{M})$, exposed to $50 \mu \mathrm{g} / \mathrm{ml}$ of red Alexa Fluor 555-conjugated transferrin (Tf-555, Molecular Probes) for 1 hour at $4^{\circ} \mathrm{C}$, washed with ice-cold PBS to remove unbound transferrin, and then cells shifted to $37^{\circ} \mathrm{C}$ for 30 minutes to allow endocytosis to occur. Uptake was terminated by washing with ice-cold PBS. Cellular membranes were stained green using DiO (Molecular Probes, 200× dilution). After washing with PBS, the cells were added onto glass coverslips coated with $0.01 \%$ poly-L-lysine and were fixed with $4 \%$ paraformaldehyde prior to mounting on glass slides using ProLong Gold Antifade Reagent containing DAPI (Life Technologies), which stains the nuclei blue, and images were obtained as previously described (37). At least 6 fields were randomly selected (approximately 100 cells), and the percentage of cells that internalized Tf-555 was calculated.

Purification of inhibitory factor(s) in G. vaginalis supernatants. The bacterial culture supernatants were filtered using Amicon centrifugal filters (EMD Millipore) with a 3-kDa cutoff, digested with $100 \mu \mathrm{g} / \mathrm{ml}$ proteinase $\mathrm{K}$ or trypsin for 1 hour at $37^{\circ} \mathrm{C}$ or heated to $56^{\circ} \mathrm{C}$ or $100^{\circ} \mathrm{C}$ for 1 hour. The $<3-\mathrm{kDa}$ fraction of $G$. vaginalis culture supernatants was dialyzed against distilled water at ambient temperature using a cellulose ester membrane with a molecular weight cutoff of $0.1-0.5 \mathrm{kDa}$ (Spectrum Labs). The aqueous layer of the dialysate and retentate were concentrated to dryness under vacuum and reconstituted in PBS to the original volume. To further purify the inhibitory compound from the dialysate $(<0.5 \mathrm{kDa})$, the bioactive fraction was further purified by HPLC equipped with a $150 \times 2.1 \mathrm{~mm}$ Cogent Diamond Hydride column (MicroSolv Technology) at a flow rate of $0.5 \mathrm{ml} / \mathrm{min}$ : 0-10 minutes, isocratic $4 \%$ water in acetonitrile; 10-25 minutes, gradient to $30 \%$ water. Fractions were collected every 3.5 minutes, and the effluent was monitored at 210 and $260 \mathrm{~nm}$. Fractions were dried in a lyophilizer and resuspended to their injection volume. The bioactive fraction was subsequently analyzed by ultra-performance liquid chromatography tandem MS (UPLC-MS/MS) (38). Fractions were separated on a Waters Acquity UPLC system in HILIC mode coupled to a Waters Synapt G2 quadrupole time-of-flight mass spectrometer, which was used to analyze the eluate.

Statistics. Analyses were performed using GraphPad Prism version 6.0 software. Results were compared using an unpaired Student's $t$ test or 1-way analysis of variance (ANOVA) with Bonferroni's post hoc test. Drug concentrations used in antiviral assays were log-transformed, and the $\mathrm{IC}_{50}$ values were calculated using nonlinear curve fitting of inhibition versus concentration data to a sigmoidal curve with a variable Hill coefficient.

Study approval. The protocol to collect vaginal swabs was approved by the Institutional Review Board, Albert Einstein College of Medicine, and participants provided written informed consent.

\section{Author contributions}

ET, S. Sinclair, and BCH wrote the manuscript. ET, S. Sinclair, PMMM, BF, MJK, DF, and BCH designed and/or supervised the study. ET, S. Sinclair, KR, SC, BW, NC, and S. Srinivasan performed the experiments. ET, S. Sinclair, PMMM, BW, and BCH analyzed the data. 


\section{Acknowledgments}

We thank Gilead, Inc. for providing TDF, TFV, and TAF. We also thank William Jacobs for use of the MS equipment. This work was supported by the NIH (NIAID grant U19AI03461).

Address correspondence to: Betsy C. Herold, Albert Einstein College of Medicine, 1225 Morris Park Avenue, Van Etten 6A03, Bronx, New York 10461, USA. Phone: 718.839.7640; Email: betsy.herold@einstein.yu.edu.

SAC's present address is: Ferrier Research Institute, Victoria University of Wellington, Lower Hutt, New Zealand.

1. Abdool Karim Q, et al. Effectiveness and safety of tenofovir gel, an antiretroviral microbicide, for the prevention of HIV infection in women. Science. 2010;329(5996):1168-1174

2. Abdool Karim SS, et al. Tenofovir gel for the prevention of herpes simplex virus type 2 infection. NEngl J Med. 2015;373(6):530-539.

3. Marrazzo JM, et al. Tenofovir-based preexposure prophylaxis for HIV infection among African women. $N$ Engl J Med. 2015;372(6):509-518

4. Rees H, et al. Conference on Retroviruses and Opportunistic Infections. Seattle, Washington; 2015. http://www.croiconference org/sessions/facts-001-phase-iii-trial-pericoital-tenofovir-1-gel-hiv-prevention-women.

5. Baeten JM, et al. Use of a vaginal ring containing dapivirine for HIV-1 prevention in women. N Engl J Med. 2016;375(22):2121-2132.

6. Nel A, et al. Safety and efficacy of a dapivirine vaginal ring for HIV prevention in women. N Engl J Med. 2016;375(22):2133-2143.

7. Van Damme L, et al. Preexposure prophylaxis for HIV infection among African women. NEngl J Med. 2012;367(5):411-422.

8. Hijazi K, et al. Expression of genes for drug transporters in the human female genital tract and modulatory effect of antiretroviral drugs. PLoS ONE. 2015;10(6):e0131405.

9. Nicol MR, et al. Expression of six drug transporters in vaginal, cervical, and colorectal tissues: Implications for drug disposition in HIV prevention. J Clin Pharmacol. 2014;54(5):574-583.

10. Taneva E, et al. Differential mechanisms of tenofovir and tenofovir disoproxil fumarate cellular transport and implications for topical preexposure prophylaxis. Antimicrob Agents Chemother. 2015;60(3):1667-1675.

11. Taneva E, et al. HIVR4P 2016. Chicago, IL: AIDS Research and Human Retroviruses; 2016:45. http://webcasts.hivr4p.org/ console/player/33023?mediaType=slideVideo\&.

12. Mesquita PM, et al. Intravaginal ring delivery of tenofovir disoproxil fumarate for prevention of HIV and herpes simplex virus infection. J Antimicrob Chemother. 2012;67(7):1730-1738.

13. Nixon B, et al. Vaginally delivered tenofovir disoproxil fumarate provides greater protection than tenofovir against genital herpes in a murine model of efficacy and safety. Antimicrob Agents Chemother. 2014;58(2):1153-1160.

14. Krakower DS, Mayer KH. Pre-exposure prophylaxis to prevent HIV infection: current status, future opportunities and challenges. Drugs. 2015;75(3):243-251.

15. Ruane PJ, et al. Antiviral activity, safety, and pharmacokinetics/pharmacodynamics of tenofovir alafenamide as 10-day monotherapy in HIV-1-positive adults. J Acquir Immune Defic Syndr. 2013;63(4):449-455.

16. Andrei G, et al. Topical tenofovir, a microbicide effective against HIV, inhibits herpes simplex virus-2 replication. Cell Host Microbe. 2011;10(4):379-389.

17. Suo Z, Johnson KA. Selective inhibition of HIV-1 reverse transcriptase by an antiviral inhibitor, (R)-9-(2-phosphonylmethoxypropyl)adenine. J Biol Chem. 1998;273(42):27250-27258.

18. Li H, Jia W. Cometabolism of microbes and host: implications for drug metabolism and drug-induced toxicity. Clin Pharmacol Ther. 2013;94(5):574-581.

19. Klatt NR, et al. Vaginal bacteria modify HIV tenofovir microbicide efficacy in African women. Science. 2017;356(6341):938-945.

20. Hillier SL, et al. Conference on Retroviruses and Opportunistic Infections. Seattle, WA; 2017. http://www.croiconference.org/ sessions/impact-vaginal-microbiota-genital-tissue-and-plasma-concentrations-tenofovir.

21. Nakra NA, et al. Loss of innate host defense following unprotected vaginal sex. J Infect Dis. 2016;213(5):840-847.

22. Murta EF, Filho AC, Barcelos AC. Relation between vaginal and endocervical pH in pre- and post-menopausal women. Arch Gynecol Obstet. 2005;272(3):211-213.

23. First episodes of genital herpes in a swedish STD population: a study of epidemiology transmission by the use of herpes simplex virus (HSV) typing specific serology. Lowhagen GB, ${ }^{\star}(1)$ Tunback P, Andersson K, Bergstrom T, Johannisson G. Sex transm infect 2000;76:179-82. Am J Ophthalmol. 2000;130(6):863.

24. Palú G, Stefanelli S, Rassu M, Parolin C, Balzarini J, De Clercq E. Cellular uptake of phosphonylmethoxyalkylpurine derivatives. Antiviral Res. 1991;16(1):115-119.

25. Lindenbaum J, Rund DG, Butler VP, Tse-Eng D, Saha JR. Inactivation of digoxin by the gut flora: reversal by antibiotic therapy N Engl J Med. 1981;305(14):789-794.

26. Ben-Dov N, Korenstein R. Enhancement of cell membrane invaginations, vesiculation and uptake of macromolecules by protonation of the cell surface. PLoS ONE. 2012;7(4):e35204.

27. Yin J, Wang J. Renal drug transporters and their significance in drug-drug interactions. Acta Pharm Sin B. 2016;6(5):363-373.

28. Chen BA, et al. Phase 1 safety, pharmacokinetics, and pharmacodynamics of dapivirine and maraviroc vaginal rings: A doubleblind randomized trial. J Acquir Immune Defic Syndr. 2015;70(3):242-249.

29. Mesquita PM, et al. Novel preclinical models of topical PrEP pharmacodynamics provide rationale for combination of drugs 
with complementary properties. Retrovirology. 2013;10:113.

30. Herold BC, et al. Impact of sex on the pharmacokinetics and pharmacodynamics of $1 \%$ tenofovir gel. Clin Infect Dis. 2016;62(3):375-382

31. Ochsenbauer C, et al. Generation of transmitted/founder HIV-1 infectious molecular clones and characterization of their replication capacity in CD4 T lymphocytes and monocyte-derived macrophages. J Virol. 2012;86(5):2715-2728

32. Nardini P, et al. Lactobacillus crispatus inhibits the infectivity of Chlamydia trachomatis elementary bodies, in vitro study. Sci Rep. 2016;6:29024.

33. Gilbert NM, Lewis WG, Lewis AL. Clinical features of bacterial vaginosis in a murine model of vaginal infection with Gardnerella vaginalis. PLoS One. 2013;8(3):e59539.

34. Kwan WS, et al. Intracellular 'boosting' of darunavir using known transport inhibitors in primary PBMC. Br J Clin Pharmacol. 2009;68(3):375-380

35. Kokkinosa A, Fasseas C, Eliopoulos E, Kalantzopoulos G. Cell size of various lactic acid bacteria as determined by scanning electron microscope and image analysis. Le Lait. 1998;78(5):491-500.

36. Fredricks DN, Fiedler TL, Thomas KK, Mitchell CM, Marrazzo JM. Changes in vaginal bacterial concentrations with intravaginal metronidazole therapy for bacterial vaginosis as assessed by quantitative PCR. J Clin Microbiol. 2009;47(3):721-726.

37. Cheshenko N, et al. HSV activates Akt to trigger calcium release and promote viral entry: novel candidate target for treatment and suppression. FASEB J. 2013;27(7):2584-2599.

38. Chandrabos $\mathrm{C}$, et al. The $\mathrm{p} 60$ and NamA autolysins from Listeria monocytogenes contribute to host colonization and induction of protective memory. Cell Microbiol. 2015;17(2):147-163. 